\title{
Magnetorotational Mechanism: Supernova Explosions and Ejections
}

\author{
N. V. Ardeljan \\ Department of computational mathematics and Cybernetics Moscow \\ State University, Vorobjevy Gory, Moscow B-234 119899, Russia, \\ E-mailardel@cs.msu.su \\ G. S. Bisnovatyi-Kogan, and S. G. Moiseenko \\ Space Research Institute, Profsoyuznaya str. 84/32 Moscow 117997 \\ Russia,E-mailgkogan@iki.rssi.ru, moiseenko@iki.rssi.ru
}

\begin{abstract}
We made simulations of the collapse of the rotating protostellar cloud. Differential rotation leads to the amplification of the toroidal component of the magnetic field and subsequent ejection of the matter due to the magnetorotational mechanism.

Our results show that at different initial configurations of the magnetic field formation of qualitatively different types of explosion takes place. Magnetic field of the dipole type produces a jet-like explosion. Quadrupole-like magnetic field produces supernova explosion whith ejection presumably near equatorial plane. Quantitative estimations of the ejected mass and energy are given.

We have done simulation of the collapse of the white dwarf and formation of a differentially rotating neutron star. After the collapse stage the rotating neutron star was formed. The rotation of the neutron star is strongly differential. The presence of the magnetic field (even the weak one) could produce magnetorotational supernova explosion.

For the simulations we have used 2D numerical scheme, based on the specially developed numerical method (conservative, implicit, triangular grid, Lagrangian, grid reconstruction).
\end{abstract}

\section{Introduction}

Magnetorotational mechanism of the supernova type II explosions was suggested by Bisnovatyi-Kogan (1970). The idea was to use magnetic fields for the transformation of the rotational energy of the star to the energy of explosion. 1D simulations of that mechanism were made by Bisnovatyi-Kogan, Popov, \& Samokhin (1976) and with more realistic magnetic fields by Ardeljan, Bisnovatyi-Kogan, $\&$ Popov (1979). In these simulations the star was represented by the infinite cylinder. 1D results were rather promising. It was shown that it is possible to produce supernova explosion by the magnetorotational mechanism. The main difficulties for the simulations of that problem in 2D and 3D are very large variation of the parameters in space (from very small densities and temperatures near 

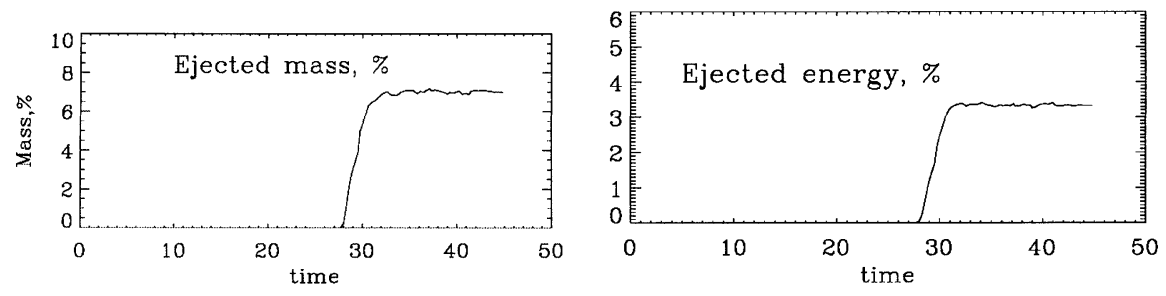

Figure 1. Time evolution of the ejected mass in $\%$ to the total mass (left), time evolution of the ejected energy in \% to the total energy (right).

the outer boundary of the star to nuclear densities and very high temperatures in the core of the star), very small magnetic fields $\left(\alpha=E_{\text {mag }} / E_{\text {grav }}=10^{6} \div 10^{-8}\right.$ where $E_{m a g}$ - initial magnetic energy, $E_{\text {grav }}$ - initial gravitational energy of the compact star. From the mathematical point of view it means that we have a problem with small parameter $\alpha$ at the time derivative in MHD equations. In such situation the application of the explicit numerical scheme is not possible due to the too strong CFL time step restriction. Another difficulty for the simulation of that problem is a strong variation of the outer boundary of the star during its evolution. In such situation the application of the ordinary numerical method would not let us get a result.

For the simulation of that problem in 2D we have used specially developed Lagrangian conservative implicit numerical method on triangular grid with the grid reconstruction (Ardeljan et al. 1996; Ardeljan, Bisnovatyi-Kogan, \& Moiseenko 2000)

\section{Simulation of the rotating magnetized cloud collapse}

As a first step for the 2D simulation of the magnetorotational supernova we have done numerical $2 \mathrm{D}$ simulation of the problem of a rotating magnetized protostellar cloud collapse .

This problem is simpler for the numerical simulation because variation of the parameters is not so strong as in case of presupernova.

After a short hydrodynamical collapse stage we got a differentially rotating configuration (rapidly rotating dense core and the oblate rarefied envelope. After that the magnetic field was "switched on". We have made simulations for different values of $\alpha=E_{\text {mag }} / E_{\text {grav }}=10^{-2} \div 10^{-6}$.

We used different initial magnetic field configurations: dipole-like and quadrupole-like ones.

The application of the quadrupole-like initial magnetic field (Ardeljan, Bisnovatyi-Kogan, \& Moiseenko 2000) led to the formation of the ejection developing predominantly near equatorial plane. As a result we have got ejection of about $7 \%$ of the total mass and $3.3 \%$ of the total energy (Fig. 1). The application of the dipole-like initial magnetic field results in the ejection along the rotational axis and can lead to the formation of the jet (Ardeljan, Bisno- 
vatyi-Kogan, $\&$ Moiseenko 2001). The first simulation with a dipole-like initial magnetic field has been made by Le Blanck \& Wilson (1970).
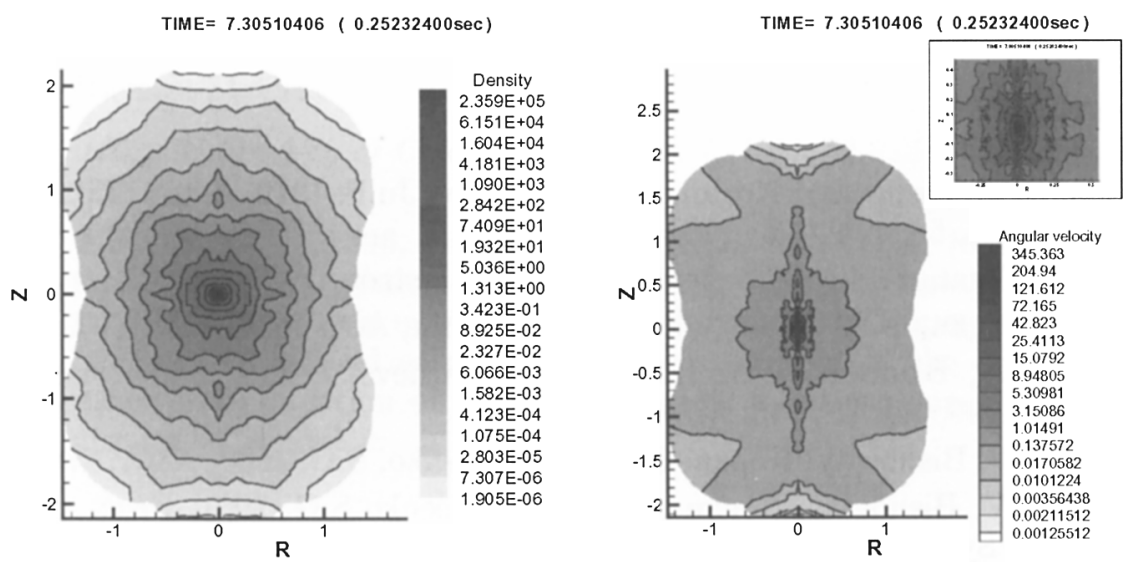

Figure 2. Density distribution (left figure) and angular velocity distribution (left figure) after the collapse of the protoneutron star.

\section{Collapse of the white dwarf and the neutron star formation}

We made simulations of the problem of the collapse of white dwarf and formation of the differentially rotating neutron star.

The initial and boundary conditions in our simulations are the same as in the paper by Ardeljan et al. (1987). We use the equation of state for supernova as in Ardeljan et al. (1987) and take into account the neutrino losses. In that paper the authors used the same numerical method, but without grid reconstruction what permitted to calculate the collapse only up to the stage of the first contraction. Application of the grid reconstruction procedure gave us the possibility to run calculations further and follow the evolution of the star through many oscillations and up to the stage of the formation of a rapidly and differentially rotating neutron star. It consists of rapidly rotating dense core and oblate slowly rotating envelope. It should be pointed out that the maximal density in the simulations of Ardeljan et al. (1987) is about $10^{5.5}$ (for about $300(!)$ grid points). We have obtained approximately the same value of the central density with about 6000 grid points.

Results of the simulations of the collapse of the white dwarf show that after the collapse the star consists of a very rapidly rotating core and slowly rotating envelope, in the transition region between core and the envelope the angular velocity is changing very rapidly. We can expect that inclusion of even a weak initial poloidal magnetic field can lead to the amplification of the toroidal component and consequent formation of the MHD shock, its amplification end supernova explosion. 
Acknowledgments. G.S.B-K. and S.G.M. would like to thank RFBR grant 02-02-16900 for the partial support and Royal Society for the support under the joint project gf/fSU/JP. S.G.M. is thankful to the organizing committee for the support and hospitality.

\section{References}

Ardeljan, N.V., Bisnovatyi-Kogan, G.S., \& Popov, Ju.P. 1979, Aston. Zh. (Sov. Astron.), 56, 1244

Bisnovatyi-Kogan, G.S., 1970, Aston. Zh. (Sov. Astron.), 47, 813

Bisnovatyi-Kogan, G.S., Popov, Ju.P., \& Samokhin, A.A. 1976 Ap\&SS, 41, 287.

Ardeljan, N.V, Bisnovatyi-Kogan, G.S., Kosmachevskii, K.V., \& Moiseenko, S.G. 1996, A\&AS, 115, 573

Ardeljan, N.V, Bisnovatyi-Kogan, G.S., \& Moiseenko, S.G. 2000, A\&A, 274, 389

Ardeljan, N.V, Bisnovatyi-Kogan, G.S., \& Moiseenko S.G. 2001, Proc. of XX Texas Symposium of Relativistic astrophysics. Austin, AIP Conf. Proc. vol. 586,439

Le Blanck, L.M. and Wilson, J.R. 1970, ApJ, 161, 541

Ardeljan, N.V., Bisnovatyi-Kogan, G.S., Popov, Yu.P. \&Chernigovskii, S.V. 1987, Astron. Zh., 64, 761 (Sov. Astron. 31, 398) 